\title{
The current temperature: a survey of post-resuscitation management across Australian and New Zealand intensive care units
}

Janet Bray, Susie Cartledge, Judith Finn, Glenn Eastwood, Nicole McKenzie, Dion Stub, Lahn Straney, Stephen Bernard Monash University, Curtin University, Alfred Hospital, Deakin University, Ambulance Victoria

\section{Background and Methods}

- Recent changes have occurred in the guideline recommendations for post resuscitation care, particularly target temperature management (TTM).

- TTM is a proven established treatment to reduce the neurological injury that occurs after cardiac arrest. However recent studies have demonstrated variation in the delivery of post-resuscitation care.

- We aimed to identify the current status of post-resuscitation practices and barriers in Australian and New Zealand ICUs.

- An online survey was sent to the Medical Directors of 163 ICUs participating in the Australian and New Zealand Intensive Care Society (ANZICS).

\section{Results}

- TTM was used in $97 \%$ of responding ICUs $(59 / 61)-64 \%$ had a TTM protocol in place.

- Majority (88\%) changed TTM practice following the TTM trial publication (NEJM 2013).

- There was wide variation in target temperatures (33-37.5C), methods for cooling and duration of cooling (12-72 hours) (Figure1).

- Half of ICU directors expressed concerns with current LOE for TTM and the Australian and New Zealand Committee on Resuscitation (ANZCOR) TTM guideline.

- Less than half $36 \%$ of ICUs had a protocol for prognostication \& treatment withdrawal.

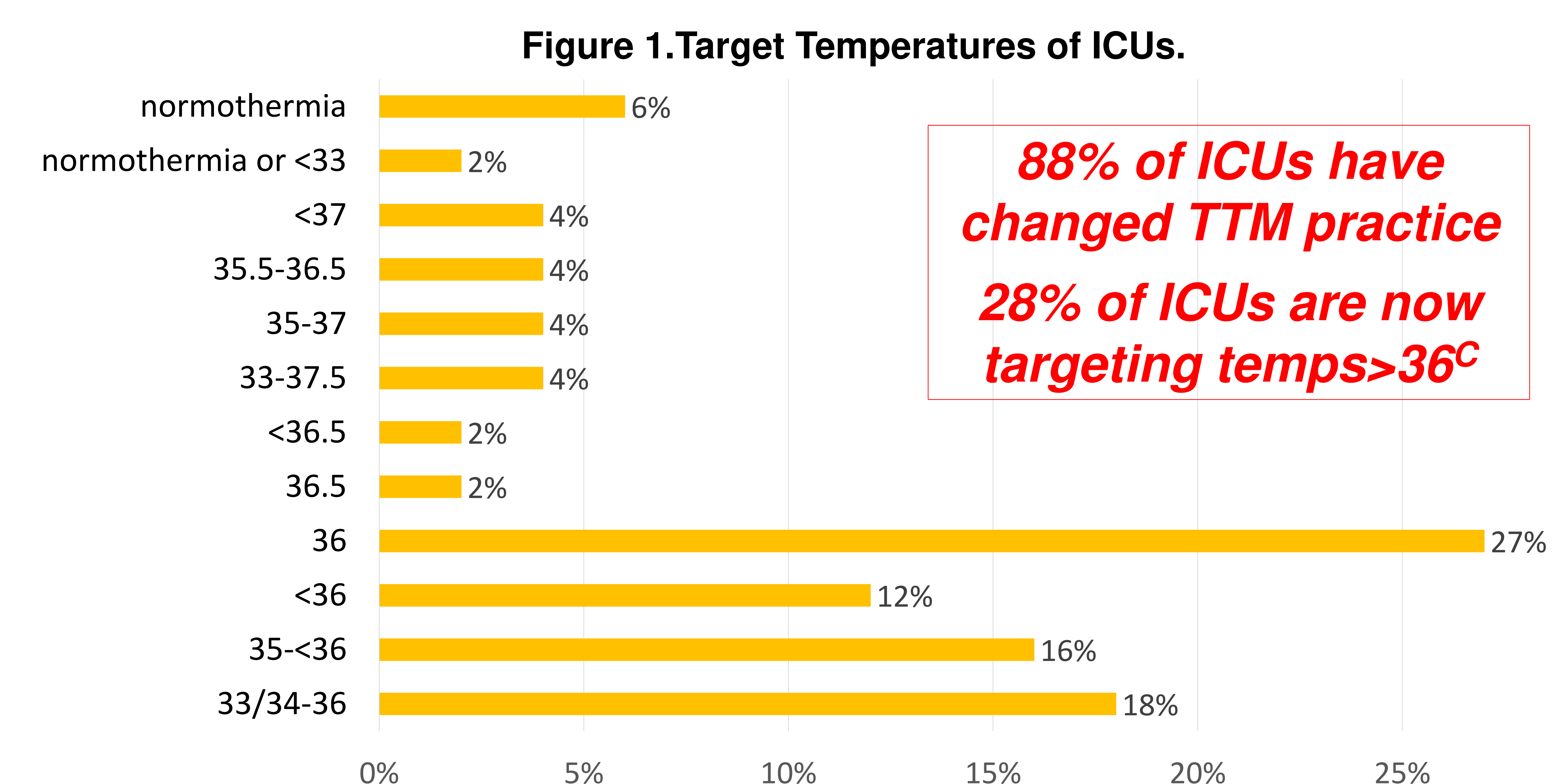

\section{Level of Evidence}
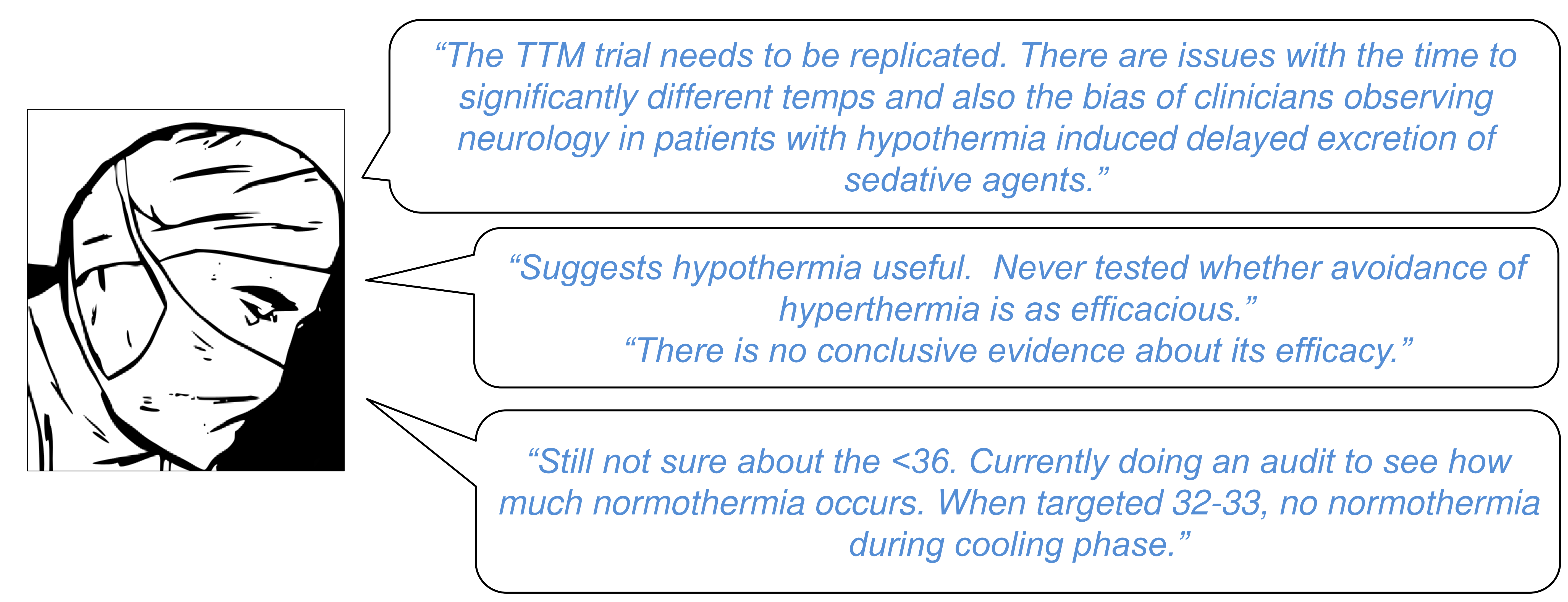

\section{ANZCOR Guideline}

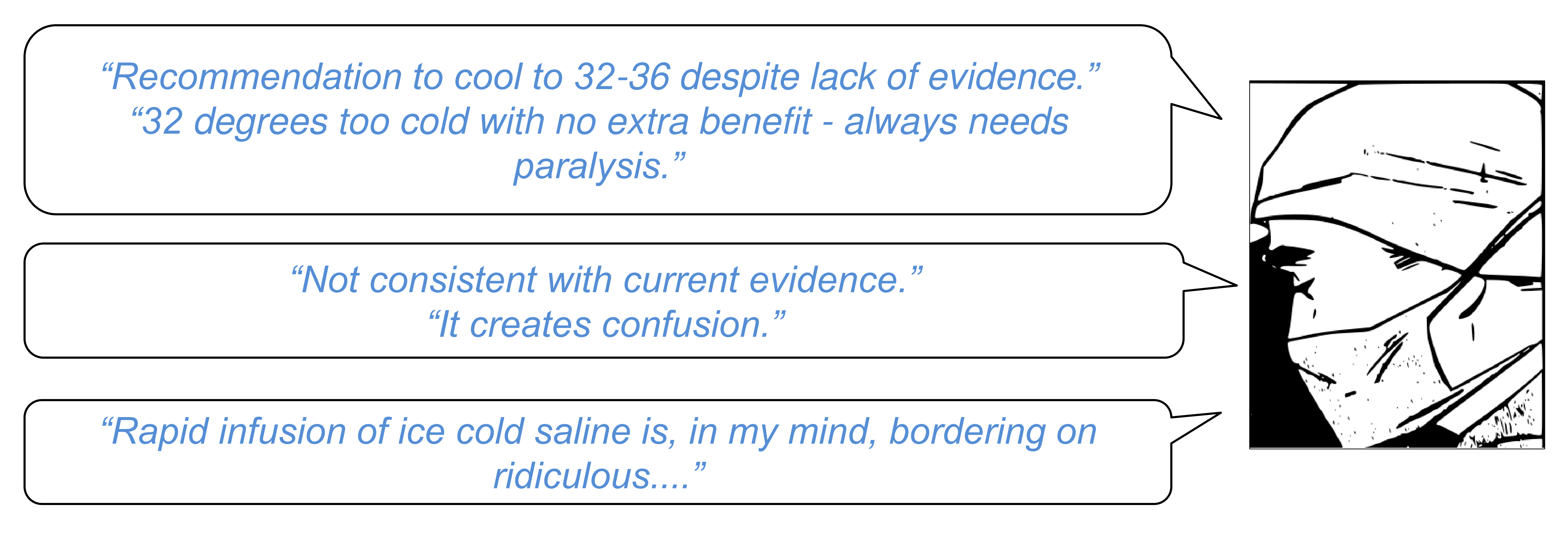

\section{Conclusions}

Post-resuscitation care varies in Australian and New Zealand ICUs. Concerns with current TTM evidence and recommendations was apparent. Some ICUs are now treating to "normothermia" - not TTM.

We intend to link this data to registries to examine the impact of TTM variation on outcomes. janet.bray@monash.edu www.ausroc.org.au
@aus_roc_@Dr_JBray 